\title{
Recurrence of atrial fibrillation after pulmonary vein isolation in dependence of arterial stiffness
}

\author{
T. Shchetynska-Marinova · M. Kranert · S. Baumann · V. Liebe · A. Grafen · S. Gerhards · S. Rosenkaimer · I. Akin · \\ M. Borggrefe · A. L. Hohneck (D)
}

Accepted: 6 September 2021 / Published online: 24 November 2021

(C) The Author(s) 2021

\begin{abstract}
Background Arterial stiffness (AS) has emerged as a strong predictor of cardiovascular $(\mathrm{CV})$ diseases. $\mathrm{Al}-$ though increased AS has been described as a predictor of atrial fibrillation (AF), its role as a risk marker for $\mathrm{AF}$ recurrence has not yet been elucidated.

Methods Patients with AF who underwent pulmonary vein isolation (PVI) were included in this study. Presence of AS was evaluated by measuring aortic distensibility $(\mathrm{AD})$ of the descending aorta by transoesophageal echocardiography.

Results In total, 151 patients (mean \pm standard deviation (SD) age $71.9 \pm 9.8$ years) were enrolled and followed for a median duration of 21 months (interquartile range 15.0-31.0). During follow-up, AF recurred in $94(62.3 \%)$ patients. AF recurrence was seen more frequently in patients with permanent AF $(27 \%$ vs $46 \%, p=0.03$ ) and in those who had undergone prior PVI ( $9 \%$ vs $23 \%, p=0.02$ ). AD was significantly reduced in patients with $\mathrm{AF}$ recurrence (mean $\pm \mathrm{SD}$ $2.6 \pm 2.3$ vs $\left.1.5 \pm 0.7 \times 10^{-3} \mathrm{~mm} \mathrm{Hg}^{-1}, p<0.0001\right)$, as well as left atrial volume index (LAVI) (mean \pm SD $29 \pm 12$ vs $\left.44 \pm 15 \mathrm{ml} / \mathrm{m}^{2}, p<0.0001\right)$. Multivariable analysis
\end{abstract}

Supplementary Information The online version of this article (https://doi.org/10.1007/s12471-021-01644-w) contains supplementary material, which is available to authorized users.

T. Shchetynska-Marinova $\cdot$ M. Kranert · S. Baumann ·

V. Liebe · A. Grafen · S. Gerhards · S. Rosenkaimer · I. Akin · M. Borggrefe · A. L. Hohneck ( $\bowtie)$

First Department of Medicine-Cardiology, University Medical Centre Mannheim, Medical Faculty Mannheim, University of Heidelberg, Mannheim, Germany annalena.hohneck@umm.de

M. Kranert · I. Akin · M. Borggrefe · A. L. Hohneck partner site Mannheim, German Centre for Cardiovascular Research (DZHK), Mannheim, Germany revealed LAVI (odds ratio (OR) 2.9, 95\% confidence interval (CI) 1.2-3.4) and AS (OR 3.6, 95\% CI 2.8-4.1) as independent risk factors of AF recurrence.

Conclusion Increased AS and left atrial size were independent predictors of $\mathrm{AF}$ recurrence after PVI. AD as surrogate marker of AS seemed to reflect the overall $\mathrm{CV}$ risk. In addition, $\mathrm{AD}$ was significantly correlated with left atrial size, which suggests that increased AS leads to atrial remodelling and thus to AF recurrence. Trial registration German registry for clinical studies (DRKS), DRKS00019007.

Keywords Atrial fibrillation · Recurrence - Arterial stiffness $\cdot$ Left atrial volume index

\section{Introduction}

Atrial fibrillation (AF) is the most commonly sustained cardiac rhythm disturbance. The prevalence of this age-related condition is still increasing $[1,2]$. Due to the large number of patients suffering from $\mathrm{AF}$ and given the limited treatment options, early detection and prevention are becoming increasingly important [3]. Although the treatment options for AF are still improving, especially interventional therapy by catheter ablation or pulmonary vein isolation (PVI), recurrences remain a major challenge.

\section{What's new?}

- In this study, arterial stiffness was not only a global parameter of cardiovascular damage, but it was also independently associated with recurrent atrial fibrillation.

- Aortic distensibility as a proxy for arterial stiffness was significantly correlated with left atrial size. 
Several predictors of recurrent AF have been identified, such as persistent AF, left atrial (LA) size or chronic kidney disease [4-7]. These predictors are also included in the APPLE score [8], a novel score for prediction of rhythm outcome. Most factors included in the APPLE score are cardiovascular (CV) components that are associated with increased AS [9]. Arterial stiffness (AS) also increases with age and is an independent predictor of CV diseases and mortality [10]. Furthermore, an association between AS and an increased risk of new-onset AF has recently been described [11, 12].

Since AS provides information on the overall CV risk, as well as on subclinical organ damage, noninvasive methods for the assessment of AS are becoming increasingly important in prevention and risk stratification [13].

Measurement of aortic distensibility (AD) as surrogate of AS, by using transoesophageal echocardiography, permits a convenient assessment, which can be easily incorporated into clinical routine of patients with AF [14]. This method could not only be time effective but is also potentially superior to conventional risk stratification. We therefore investigated the role of $\mathrm{AD}$, as a surrogate of increased $\mathrm{AS}$ and overall $\mathrm{CV}$ risk, in the prediction of recurrence of AF after PVI.

\section{Methods}

\section{Study population}

This monocentric observational trial was conducted at the First Department of Medicine (Cardiology), University Medical Centre Mannheim in Mannheim, Germany. Patients with AF who underwent PVI were included in the study. Written informed consent was obtained from all patients. Recruitment started in June 2015 and ended in December 2017. Patients were followed prospectively. Other results of this study population have been reported previously [6].

Baseline demographic and clinical characteristics were acquired from a questionnaire and patient charts. Exclusion criteria at the time of transoesophageal echocardiography were acute myocardial infarction or stroke (within past 30 days), indication for aortocoronary bypass operation, minimal heart rate at rest $<50 \mathrm{bpm}$, need for pacemaker stimulation, hypotension with blood pressure $<90 / 50 \mathrm{~mm} \mathrm{Hg}$ or uncontrolled hypertension with systolic blood pressure $\geq 160 \mathrm{mmHg}$, history of aortic dissection/ aneurysm and history of aortic prosthesis.

The study was conducted according to the principles of the Declaration of Helsinki and was approved by the local ethics committee, the Medical Ethics Commission II, Faculty of Medicine Mannheim, University of Heidelberg, Germany. Data were analysed anonymously. Data protection was in accordance with the European Union's Data Protection Directive.

\section{Assessment of atrial fibrillation}

Recurrence of AF was based on electrocardiographic evidence, including 72-hour Holter monitoring. Classification of AF was performed in accordance with the current European Society of Cardiology (ESC) guidelines for the management of atrial fibrillation [15], whereby two patterns-paroxysmal and persistent $\mathrm{AF}$ - were distinguished.

Prior to PVI, clinical risk scores for prediction of thromboembolism $\left(\mathrm{CHA}_{2} \mathrm{DS}_{2}\right.$-VASc score: congestive heart failure or left ventricular systolic dysfunction, hypertension, age $\geq 75$ years, diabetes mellitus, prior stroke or transient ischaemic attack, vascular disease, age 65-74 years, female sex [16]), rhythm outcome after ablation (APPLE score: age $>65$ years, persistent AF, impaired estimated glomerular filtration rate (eGFR) $<60 \mathrm{ml} / \mathrm{min}$ per $1.73 \mathrm{~m}^{2}$, LA diameter $\geq 43 \mathrm{~mm}$, ejection fraction $<50 \%$ [8]) and AF symptom classification (European Heart Rhythm Association score [17]) were assessed.

\section{Echocardiography}

Transthoracic and transoesophageal echocardiographic examinations were performed at the time of study inclusion using Vivid E9 (GE Vingmed Ultrasound, Horten, Norway) or iE33 (Philips Medical Systems, Andover, MA, USA).

Cardiac chamber size and function were assessed using standard M-mode, 2-dimensional (2D) and colour Doppler imaging [18]. Left ventricular ejection fraction (LVEF) was calculated using Simpson's method. LA measurements were performed at endsystole in the apical two-chamber (2Ch) and fourchamber (4Ch) views. Echocardiographic measurements of diastolic function were performed according to the recommendation for the evaluation of LV diastolic function by echocardiography [19].

The descending aorta was routinely imaged at the end of each assessment. Cross-sectional systolic and diastolic aortic diameters were measured with an electrocardiogram as a guide using $2 \mathrm{D}$ imaging techniques [20]. If echocardiography was performed for AF, measurements from the available heartbeats were averaged (usually over 3 to 5 cardiac cycles). If available, measurements were performed at multiple levels of the descending aorta. Brachial blood pressures were measured at the time of transoesophageal echocardiography using standardised protocols.

$\mathrm{AD}$ was calculated using the aortic diameters and brachial blood pressures as follows [21]:

$$
\mathrm{AD}=(\mathrm{AoSD}-\mathrm{AoDD}) / \mathrm{AoDD} \times(\mathrm{SBP}-\mathrm{DBP})
$$

AoSD aortic systolic diameter, $A o D D$ aortic diastolic diameter, $S B P$ systolic blood pressure, $D B P$ diastolic blood pressure. 


\section{Reproducibility}

To ensure reproducibility of $\mathrm{AD}$ assessment, a randomly generated set of 40 transoesophageal echocardiographic scans was analysed before study enrolment by one single experienced observer (TS-M), who was blinded to the data. Intraobserver variability for $\mathrm{AD}$ was determined using the calculation of intraclass correlation coefficient (ICC).

\section{Pulmonary vein isolation}

A diagnostic quadripolar electrode catheter (EP Technologies Inc., Sunnyvale, CA, USA) was positioned in the coronary sinus with a femoral vein approach. Transseptal catheterisation was performed, and systemic anticoagulation was achieved with intravenous heparin to maintain an activated clotting time of 250-350s.

A multipolar Lasso catheter (Biosense Webster Inc., Irvine, CA, USA) was placed into the left atrium to map signals at the ostial sides of the pulmonary veins. A deflectable, quadripolar 7-Fr catheter with 2-5-2$\mathrm{mm}$ interelectrode spacing and a $4-\mathrm{mm}$ distal electrode with an embedded thermistor (EP Technologies Inc., Sunnyvale, CA, USA) was inserted into the left atrium, either through the same transseptal puncture site or through a second transseptal puncture, and was used for ablation. Bipolar and unipolar electrograms were filtered at bandpass settings of $30-500 \mathrm{~Hz}$ and $0.05-200 \mathrm{~Hz}$, respectively, and were recorded digitally (EPMed Systems Inc., Mount Arlington, NJ, USA).

Pacing was performed from the coronary sinus or left atrium with a stimulator (Model EP-3 Clinical Stimulator, EPMed Systems Inc., Mount Arlington, NJ, USA). Complete isolation was verified as a reduction of all signals $\leq 0.2 \mathrm{mV}$ and by pacing manoeuvres.

\section{Primary endpoint}

Recurrence of AF after PVI, based on electrocardiographic evidence, served as primary endpoint.

\section{Follow-up}

Patients with AF were followed up for a median duration (interquartile, IQR) of 21 months (15.0-31.0). Periodical follow-up was performed during routine visits in our outpatient clinic. Three months after PVI, 72hour Holter monitoring was performed.

\section{Statistical analysis}

Data are presented as mean \pm standard deviation, median (IQR) or frequency (percentage). Continuous variables were compared using a two-tailed Student's $t$-test for parametric and Mann-Whitney $U$ test for nonparametric variables. Categorical variables were compared with a $\chi^{2}$ test.
Correlation analysis was performed with univariate linear regression analysis to test associations between $\mathrm{AD}$ as dependent variable and several clinical characteristics. Multivariable analysis with logistic regression analysis was performed using block entry of variables with $p<0.01$ in univariate analysis. There variables were: eGFR $\left(\mathrm{ml} / \mathrm{min}\right.$ per $\left.1.73 \mathrm{~m}^{2}\right)$, heart failure, treatment with amiodarone, $\mathrm{CHA}_{2} \mathrm{DS}_{2}$-VASc score, APPLE score, left atrial volume index (LAVI) (in $\mathrm{ml} / \mathrm{m}^{2}$ ) and $\mathrm{AD}$. ICC was calculated to assess intraobserver variability for $\mathrm{AD}$, providing a coefficient ranging from 0 to 1 and its $95 \%$ confidence interval (CI), with an ICC close to 1 indicating high similarity.

For all statistical analyses, $p<0.05$ was considered statistically significant. All statistical analyses were performed with Statistical 1 Package for Social Sciences (SPSS for Windows 24.0, Chicago, IL, USA) or GraphPad Prism 8.0 (GraphPad Software Inc., San Diego, CA, USA).

\section{Results}

\section{Baseline}

In total, 151 patients were included in the study (mean age $71.9 \pm 9.8$ years, $64 \%$ male). The patients suffered from several cardiovascular comorbidities, such as hypertension (78\%), diabetes mellitus (13\%), chronic kidney disease (32\%), coronary artery disease (29\%), heart failure $(43 \%)$ and obstructive sleep apnoea $(13 \%)$. Stroke or transitory ischaemic attack had occurred in $12 \%$ of the patients and $18 \%$ had a history of smoking.

Concomitant medical treatment was in accordance with the 2016 ESC Guidelines for the management and treatment of atrial fibrillation [15]; 72\% of the patients were taking a betablocker, $54 \%$ an angiotensinconverting enzyme inhibitor or angiotensin receptor blocker and $19 \%$ a calcium channel blocker. Statin use was observed in $41 \%$ of the patients and $7 \%$ received diabetes medication. A small proportion was on antiarrhythmic treatment with amiodarone (11\%) or digitalis $(9 \%)$.

Persistent AF was diagnosed in 59 patients (39\%). Mean $\mathrm{CHA}_{2} \mathrm{DS}_{2}$-VASc score was $3.0 \pm 1.7$, while mean APPLE score was $1.9 \pm 1.2$. Prior PVI had been performed in 27 patients (18\%). Hundred and thirty-five patients $(89 \%)$ received anticoagulant therapy, consisting of vitamin $\mathrm{K}$ antagonists or direct oral anticoagulants.

Most patients had a normal systolic cardiac function (LVEF 56 $\pm 9 \%$ ); impaired LVEF $(<50 \%)$ was found in 25 patients $(27 \%)$. Mean LA dimensions, both LA diameter measured by M-mode $(42 \pm 7 \mathrm{~mm})$ and LAVI $\left(40 \pm 16 \mathrm{ml} / \mathrm{m}^{2}\right)$ were elevated and outside of the normal range [18, 22]. Mean $\mathrm{AD}$ was reduced $\left(1.9 \pm 1.1 \times 10^{-3} \mathrm{~mm} \mathrm{Hg}^{-1}\right)$ compared with normal values [23]. 
Demographics, baseline characteristics and drug treatment specifics of the study population are shown in Tab. S1 in the Electronic Supplementary Material.

\section{Follow-up}

During follow-up, AF recurred in 94 patients (62.3\%). We subsequently divided the cohort into two groups: patients without or with recurrent AF. Vital parameters (heart rate, blood pressure and pulse pressure) were not different between the groups. Patients with recurrent $\mathrm{AF}$ more often had hypertension $(66 \%$ vs $83 \%, p=0.04$ ), heart failure ( $24 \%$ vs $54 \%, p<0.0001$ ) and a higher $\mathrm{CHA}_{2} \mathrm{DS}_{2}$-VASc score $(2.5 \pm 1.7$ vs $3.3 \pm 1.6$, $p=0.004)$. Chronic kidney disease was also more common in patients with $\mathrm{AF}$ recurrence $(17.5 \%$ vs $40.4 \%$, $p=0.003)$, with significantly lower eGFR values $(62 \pm 14$ vs $56 \pm 14 \mathrm{ml} / \mathrm{min}$ per $1.73 \mathrm{~m}^{2}, p=0.009$ ). The presence of CV risk factors, such as nicotine consumption or diabetes, was similar. There were no differences regarding concomitant treatment with antihypertensive drugs, statins, diabetes medication or anticoagulant therapy. In contrast, treatment with amiodarone ( $1.8 \%$ vs $16.0 \%, p<0.01)$ or digitalis $(1.8 \%$ vs $13.8 \%$, $p=0.02$ ) was more common in the recurrence group. $\mathrm{AF}$ recurred more often in patients with persistent $\mathrm{AF}$ ( $27 \%$ vs $46 \%, p=0.03)$ and in those who had undergone prior PVI ( $9 \%$ vs $23 \%, p=0.02)$. Patients with recurrent AF had a significantly higher APPLE score $(1.4 \pm 1.0$ vs $2.2 \pm 1.2, p<0.0001)$.

LVEF was lower in patients with AF recurrence ( $58 \pm 7$ vs $54 \pm 10 \%, p=0.02$ ), with a higher proportion of patients with impaired LVEF in the recurrence group (9\% vs $21 \%, p=0.04$ ). LA dimensions and volumes were significantly higher in patients with recurrent $\mathrm{AF}$ (LA M-mode $40 \pm 6$ vs $43 \pm 7 \mathrm{~mm}, p=0.01$; LAVI $29 \pm 12$ vs $\left.44 \pm 15 \mathrm{ml} / \mathrm{m}^{2}, p<0.0001\right)$. AD was significantly reduced in patients with $\mathrm{AF}$ recurrence (2.6 \pm 2.3 vs $\left.1.5 \pm 0.7 \times 10^{-3} \mathrm{~mm} \mathrm{Hg}^{-1}, p<0.0001\right)$ (Fig. 1). ICC was calculated for the assessment of intraobserver variability of $\mathrm{AD}$ and showed excellent to nearly perfect agreement (0.92, 0.81-0.96). Complete results are displayed in Tab. S1 (see Electronic Supplementary Material).

Variables with $p<0.01$ in univariate analysis were then studied in multivariable analysis, which revealed LAVI (odds ratio (OR) 2.9, 95\% CI 1.2-3.4) and AS (OR 3.6, 95\% CI 2.8-4.1) were independent risk factors for $\mathrm{AF}$ recurrence. The other variables failed to reach statistical significance (Tab. S2 in the Electronic Supplementary Material).

To determine the potential significance of $A D$ as a surrogate of overall CV risk, correlation analysis was performed, with $\mathrm{AD}$ as the dependent variable. $\mathrm{AD}$ was significantly correlated with age $(\mathrm{r}=-0.23$, $p=0.005)$, hypertension $(\mathrm{r}=-0.27, p=0.0001)$, as well as systolic blood pressure $(\mathrm{r}=-0.19, \quad p=0.02)$ and pulse pressure $(\mathrm{r}=-0.24, p=0.004)$, eGFR $(\mathrm{r}=0.31$, $p=0.0001)$, heart failure $(\mathrm{r}=-0.26, p=0.002)$ and

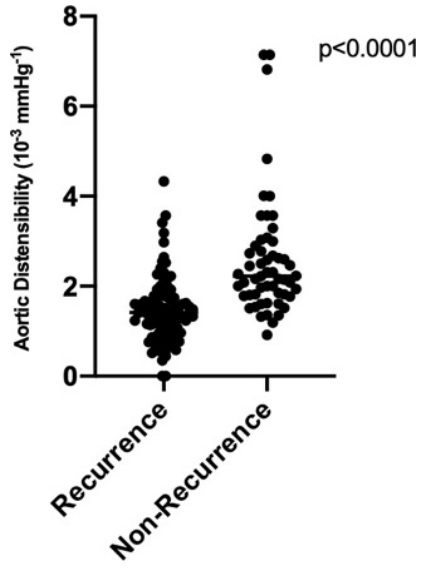

Fig. 1 Recurrence of atrial fibrillation in dependence of aortic distensibility

Table 1 Correlation analysis of $A D$ and clinical characteristics

\begin{tabular}{|l|ll}
\hline Variable & $\mathrm{r}(95 \% \mathrm{Cl})$ & $P$-value \\
\hline Age, years & $-0.23(-0.43$ to -0.13$)$ & 0.005 \\
\hline Systolic blood pressure, $\mathrm{mm} \mathrm{Hg}$ & $-0.19(-0.34$ to -0.02$)$ & 0.02 \\
\hline Pulse pressure, $\mathrm{mm} \mathrm{Hg}$ & $-0.24(-0.38$ to -0.08$)$ & 0.004 \\
\hline Hypertension & $-0.27(-0.41$ to -0.11$)$ & 0.001 \\
\hline eGFR, $\mathrm{ml} /$ min per $1.73 \mathrm{~m}^{2}$ & $0.31(0.16$ to 0.45$)$ & 0.0001 \\
\hline Three-vessel disease & $-0.17(-0.33$ to -0.01$)$ & 0.03 \\
\hline Heart failure & $-0.26(-0.41$ to -0.09$)$ & 0.002 \\
\hline $\mathrm{CHA}_{2} \mathrm{DS} 2-\mathrm{VASc}$ score & $-0.25(-0.40$ to -0.09$)$ & 0.002 \\
\hline VKA use & $-0.18(-0.33$ to -0.01$)$ & 0.03 \\
\hline LVEF, \% & $0.18(0.02$ to 0.34$)$ & 0.03 \\
\hline LA M-mode, mm & $-0.22(-0.41$ to -0.01$)$ & 0.04 \\
\hline LA volume $4 \mathrm{Ch}, \mathrm{ml}$ & $-0.31(-0.47$ to -0.11$)$ & 0.002 \\
\hline LAVI, ml/m ${ }^{2}$ & $-0.36(-0.52$ to -0.17$)$ & 0.0003 \\
\hline
\end{tabular}

Cl confidence interval, eGFR estimated glomerular filtration rate, VKA vitamin $\mathrm{K}$ antagonist, $L V E F$ left ventricular ejection fraction, $L A$ left atrial, 4 Ch four-chamber view, LAVI left atrial volume index

$\mathrm{CHA}_{2} \mathrm{DS}_{2}$-VASc score $(\mathrm{r}=-0.25, p=0.002)$. Coronary three-vessel disease $(\mathrm{r}=-0.17, p=0.03)$, use of vitamin $\mathrm{K}$ antagonists $(\mathrm{r}=-0.18, p=0.03)$ and LVEF $(\mathrm{r}=0.18, p=0.03)$ showed less strong associations. In addition, a significant inverse correlation was seen for LA dimensions (LA M-mode, $r=-0.22, p=0.04$ ) and LA volumes (LA volume 4Ch: $\mathrm{r}=-0.31, p=0.002$; LAVI: $\mathrm{r}=-0.36, p=0.0003$ ) (Tab. 1).

\section{Discussion}

In the present study, we explored the predictive role of AS in AF recurrence after PVI. The results of our analysis showed that patients with decreased $\mathrm{AD}$ were more likely to have recurrences of AF. Besides AD, LAVI remained an independent predictor after multivariable analysis.

In recent years, several studies have demonstrated the impact of various clinical parameters to predict AF recurrence. Generally, it is recognised that per- 
manent AF, LA size, hypertension, diabetes mellitus, obstructive sleep apnoea, heart failure, and early AF recurrence after PVI [24-29] are associated with recurrent $\mathrm{AF}$, which is why multimorbid patients are more likely to be considered at risk of recurrence.

AF type and severity of symptoms are the most wellestablished parameters when selecting a rhythm control strategy [30]. There is strong evidence showing that persistent $\mathrm{AF}$ is associated with a higher recurrence rate and a higher stroke and mortality risk than paroxysmal AF [30]. Our data support these previously reported findings, with an increase in AF recurrence in patients with persistent AF compared with those with paroxysmal AF. However, in our study, the AF phenotype lost its predictive value when adjusting for other predictors in multivariable analysis. A possible explanation is that not the AF phenotype itself but the underlying comorbidities and possibly the level of atrial fibrosis and inflammation, as shown by Marrouche et al., are the main risk factors for recurrences of $\mathrm{AF}$ [31].

In recent years, AS has emerged as a strong predictor of CV disease, end-organ damage and all-cause mortality. Moreover, several clinical studies have reported that AS is associated with new-onset $\mathrm{AF}$ [32-34], as well as with AF recurrence after initially successful restoration of sinus rhythm [35-38]. It is recognised that the stiffening of large arteries and the aorta may exert severe effects on CV function, including cardiac remodelling and diastolic dysfunction, systolic hypertension, and reduced coronary perfusion $[39,40]$. Furthermore, excessive pressure pulsatility may contribute to microvascular dysfunction, leading to end-organ damage [39]. Given that increased AS may be present long before the first clinical manifestations of adverse haemodynamics and end-organ damage appear [41, 42], AS can be used as a valid indicator of the likelihood of success of attempts at rhythm control and thus influence clinical decision-making to choose the best treatment option in patients with AF. Especially in patients who are considered to have 'a low risk of recurrence', due to the absence of conventional risk factors, measuring $\mathrm{AD}$ could provide earlier information on structural alterations that are also associated with an increased risk of recurrence.

In our study, $\mathrm{AD}$ was significantly reduced in patients with recurrent AF, reflecting increased AS. Few previous reports, which used the cardio-ankle vascular index and peripheral pulse wave velocity as surrogate measures of arterial stiffness, enforce our results $[36,43,44]$. Fumagalli et al. demonstrated that the presence of AF during follow-up is directly correlated with AS, as assessed by the cardio-ankle vascular index [44]. Lau et al. found that patients with the highest levels ( $\geq 75$ th percentile) of peripheral pulse pressure, central pulse pressure and augmentation pressure have higher recurrence rates of lone $\mathrm{AF}$ [36]. In contrast, Kizilirmak et al. did not find an independent association between parameters of AS and AF recur- rence and concluded that LA size is the strongest independent predictor of AF recurrence [37]. Although the matter remains controversial, which may also be due to the lack of standardisation in the assessment of AS, the preposition that AS can be used as a valid predictor of AF recurrence is pathophysiologically plausible.

Several mechanisms may explain the association between AS and AF. Increased AS and its pathological haemodynamic pattern may be associated with increased afterload, decreased LV compliance, and subsequent LA remodelling and enlargement, which may represent a substrate for development maintenance of AF [45]. Furthermore, increased AS results in excessive pulsatility in the aorta, which is transmitted preferentially to low-resistance vascular beds (such as the kidney, liver and brain) and causes end-organ damage. These events may worsen the comorbidity state in AF patients, which could also contribute to AF progression. Thus, considering the adverse haemodynamic effects of AS, its predictive role in AF recurrence is not surprising.

Interestingly, the possible consequences of increased AS discussed above (chronic kidney disease, hypertension, heart failure) were also significantly associated with recurrences of AF in our study. However, it cannot be ascertained whether increased AS is an independent risk factor for the progression of AF or a global marker of overall CV risk and end-organ dysfunction. On the other hand, AF itself could promote negative vascular changes by promoting atherosclerosis and inflammation. Therefore, we believe that both increased AS and AF could promote each other, forming a vicious circle of adverse haemodynamic, structural and functional CV changes.

Since the LA diameter does not reflect the correct size of the left atrium, measuring LA volume is a more correct approach [46]. For this reason, LAVI has been increasingly used as a proxy for LA size in clinical trials. The risk of AF recurrence increases with the size of the left atrium [47], whereby a cut-off value $>34 \mathrm{ml} / \mathrm{m}^{2}$ is seen in several studies $[6,48]$. In the current study, LAVI was identified as an independent predictor of AF recurrence. Furthermore, we found a significant correlation of LAVI with AD. This is in accordance with the literature.

In hypertensive patients, AS is significantly correlated with LA size [49], which leads to the assumption that AS is not only a surrogate for CV risk but directly promotes structural cardiac changes [50]. These adverse effects appear to be reversible, to a certain extent. This has been demonstrated by Tsang et al., who showed that treatment with quinapril leads to a decrease in LAVI and an improvement in AS [51]. AS could therefore be the missing link, leading to recurrent AF through atrial remodelling.

For this reason, AS could be an interesting therapeutic target to break the vicious circle and to help identify patients at higher risk. $\mathrm{AD}$, in contrast to LAVI, could be an early marker because increased LAVI indi- 


\section{Advertisement placed here.}

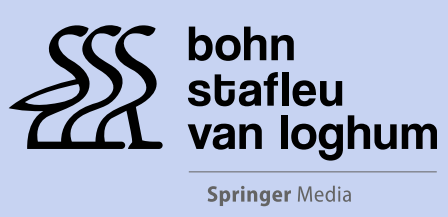

Houten 2021 


\section{Advertisement placed here.}

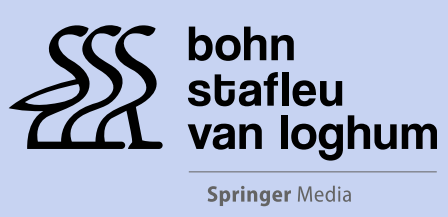

Houten 2021 
cates the terminal stage of cardiac remodelling, while AS reflects an ongoing process. On the other hand, $\mathrm{AD}$ is not a specific parameter and is influenced by various factors. In our cohort, $\mathrm{AD}$ was significantly correlated with age, blood pressure, kidney function, heart failure and LA size. Scoring systems such as the $\mathrm{CHA}_{2} \mathrm{DS}_{2}$-VASc score and APPLE score are composed of many of these parameters, which are used to assess the risk of $\mathrm{AF}$ recurrence. The implementation of $\mathrm{AD}$ in risk stratification of patients with $\mathrm{AF}$ could thus provide a simplified approach to reduce these complex scoring systems to a single value. Since AS may predict the risk of $\mathrm{AF}$ recurrence independently of traditional risk factors and is also likely to predict overall CV risk, its implementation in clinical practice could lead to both time savings and better prediction. Compared with established factors such as LAVI, AD was not inferior.

\section{Study limitations}

Our study population consisted of patients with complicated AF who were treated at a university hospital and not by a general physician, which may have led to a higher proportion of AF recurrences. On the other hand, especially in asymptomatic patients, the incidence of recurrent AF may have been underestimated. Even though Holter monitoring was performed periodically, paroxysmal episodes may not have been detected.

\section{Conclusion}

The current study evaluated the predictive role of AS in AF recurrence. Both AS, measured as $\mathrm{AD}$, and $\mathrm{LA}$ size were independently positively associated with $\mathrm{AF}$ recurrence during follow-up. In addition, $\mathrm{AD}$ was significantly correlated with LA size, which leads to the assumption that increased AS promotes cardiac remodelling and hence maintains AF. For this reason, the use of $\mathrm{AD}$ measurements may help in the evaluation of the individual risk of AF recurrence after rhythm control therapy. However, further randomised clinical trials are needed to prove whether risk prediction by evaluating $\mathrm{AD}$ is not only time effective but also more accurate than conventional risk stratification. Our results should be verified in a larger and less complex patient population, in order to derive a recommendation for clinical practice.

Conflict of interest T. Shchetynska-Marinova, M. Kranert, S. Baumann, V. Liebe, A. Grafen, S. Gerhards, S. Rosenkaimer, I. Akin, M. Borggrefe and A.L. Hohneck declare that they have no competing interests.

Open Access This article is licensed under a Creative Commons Attribution 4.0 International License, which permits use, sharing, adaptation, distribution and reproduction in any medium or format, as long as you give appropriate credit to the original author(s) and the source, provide a link to the Creative Commons licence, and indicate if changes were made. The images or other third party material in this article are included in the article's Creative Commons licence, unless indicated otherwise in a credit line to the material. If material is not included in the article's Creative Commons licence and your intended use is not permitted by statutory regulation or exceeds the permitted use, you will need to obtain permission directly from the copyright holder. To view a copy of this licence, visit http://creativecommons.org/licenses/by/4.0/.

\section{References}

1. Kannel WB, Wolf PA, Benjamin EJ, Levy D. Prevalence, incidence, prognosis, and predisposing conditions for atrial fibrillation: population-based estimates 1. Am J Cardiol. 1998:82:2N-9N.

2. Heeringa J, van der Kuip DA, Hofman A, et al. Prevalence, incidence and lifetime risk of atrial fibrillation: the Rotterdam study. Eur Heart J. 2006;27:949-53.

3. Benjamin EJ, Chen P-S, Bild DE, et al. Prevention of atrial fibrillation: report from a national heart, lung, and blood institute workshop. Circulation. 2009;119:606-18.

4. Balk EM, Garlitski AC, Alsheikh-Ali AA, Terasawa T, Chung M, Ip S. Predictors of atrial fibrillation recurrence after radiofrequency catheter ablation: a systematic review. JCardiovasc Electrophysiol. 2010;21:1208-16.

5. Zhuang J, Wang Y, Tang K, et al. Association between left atrial size and atrial fibrillation recurrence after single circumferential pulmonary vein isolation: a systematic review and meta-analysis of observational studies. Europace. 2012;14:638-45.

6. Kranert M, Shchetynska-Marinova T, Liebe V, et al. Recurrence of atrialfibrillation in dependence ofleft atrialvolume index. In Vivo. 2020;34:889-96.

7. Li M, Liu T, Luo D, Li G. Systematic review and metaanalysis of chronic kidney disease as predictor of atrial fibrillation recurrence following catheter ablation. Cardiol J. 2014;21:89-95.

8. Kornej J, Hindricks G, Shoemaker MB, et al. The APPLE score: a novel and simple score for the prediction of rhythm outcomes after catheter ablation of atrial fibrillation. Clin Res Cardiol. 2015;104:871-6.

9. Laurent S, Boutouyrie P. Arterial stiffness: a new surrogate end point for cardiovascular disease? J Nephrol. 2007;20:S45-50.

10. Vlachopoulos C, Aznaouridis K, Stefanadis C. Prediction of cardiovascular events and all-cause mortality with arterial stiffness: a systematic review and meta-analysis. Am J Cardiol. 2010;55:1318-27.

11. Cremer A, Lainé M, Papaioannou G, Yeim S, Gosse P. Increased arterial stiffness is an independent predictor of atrial fibrillation in hypertensive patients. J Hypertens. 2015;33:2150-5.

12. Shaikh AY, Wang N, Yin X, et al. Relations of arterial stiffness and brachial flow-mediated dilation with new-onset atrial fibrillation: the Framingham heart study. Hypertension. 2016;68:590-6.

13. Williams B, Mancia G, Spiering W, et al. 2018 ESC/ESH guidelines for the management of arterial hypertension: the task force for the management of arterial hypertension of the European society of cardiology (ESC) and the European society of hypertension (ESH). Eur Heart J. 2018;39:3021-104.

14. Lang RM, Cholley BP, Korcarz C, Marcus RH, Shroff SG. Measurement of regional elastic properties of the human aorta. A new application of transesophageal echocardiography with automated border detection and calibrated subclavian pulse tracings. Circulation. 1994;90:1875-82. 
15. Kirchhof P, Benussi S, Kotecha D, et al. 2016 ESC guidelines for the management of atrial fibrillation developed in collaboration with EACTS. Eur HeartJ. 2016;37:2893-962.

16. Boriani G, Botto GL, Padeletti L, et al. Improving stroke risk stratification using the CHADS2 and CHA2DS2-VASc risk scores in patients with paroxysmal atrial fibrillation by continuous arrhythmia burden monitoring. Stroke. 2011;42:1768-70.

17. Wynn GJ, Todd DM, Webber M, et al. The European heart rhythm association symptom classification for atrial fibrillation: validation and improvement through a simple modification. Europace. 2014;16:965-72.

18. Lang RM, Badano LP, Mor-Avi V, et al. Recommendations for cardiac chamber quantification by echocardiography in adults: an update from the American society of echocardiography and the European association of cardiovascular imaging. Eur Heart J Cardiovasc Imaging. 2015;16:233-71.

19. Nagueh SF, Smiseth OA, Appleton CP, et al. Recommendations for the evaluation of left ventricular diastolic function by echocardiography: an update from the American society of echocardiography and the European association of cardiovascular imaging. Eur J Echocardiogr. 2016;17:1321-60.

20. Whitlock MC, Hundley WG. Noninvasive imaging of flow and vascular function in disease of the aorta. JACC Cardiovasc Imaging. 2015;8:1094-106.

21. Go OD, Safar ME, Smulyan H. Assessment of aortic stiffness by transesophageal echocardiography. Echocardiography. 2014;31:1105-12.

22. Nagueh SF, Smiseth OA, Appleton CP, et al. Recommendations for the evaluation of left ventricular diastolic function by echocardiography: an update from the American society of echocardiography and the European association of cardiovascular imaging. J Am Soc Echocardiogr. 2016;29:277-314.

23. Nemes A, Forster T, Lengyel C, Csanády M. Reduced aortic distensibility and coronary flow velocity reserve in diabetes mellitus patients with a negative coronary angiogram. Can J Cardiol. 2007;23:445-50.

24. Takigawa M, Takahashi A, Kuwahara T, et al. Long-term follow-up after catheter ablation of paroxysmal atrial fibrillation: the incidence of recurrence and progression of atrial fibrillation. Circ Arrhythm Electrophysiol. 2014;7:267-73.

25. Vlachos K, Letsas KP, Korantzopoulos P, et al. Prediction of atrial fibrillation development and progression: current perspectives. World J Cardiol. 2016;8:267.

26. Kochhäuser S, Dechering DG, TroughtK, etal. Predictors for progression of atrial fibrillation in patients awaiting atrial fibrillation ablation. Can J Cardiol. 2016;32:1348-54.

27. Quan D, Huang H, Kong B, Li Q, Liao J, Wang G. Predictors of late atrial fibrillation recurrence after cryoballoon-based pulmonary vein isolation: a meta-analysis. Kardiol Pol. 2017;75:376-85.

28. Lewicka E. Prediction of recurrence after cryoballoon ablation therapy in patients with paroxysmal atrial fibrillation. Anatol J Cardiol. 2016;16:489-90.

29. Johner N, Namdar M, Shah DC. Individualised approaches for catheter ablation of AF: patient selection and procedural endpoints. Arrhythm Electrophysiol Rev. 2019;8:184.

30. Agewall S, Camm J. New ESC/EACTS guidelines for the management of atrial fibrillation. : Oxford University Press; 2017.

31. Marrouche NF, Wilber D, Hindricks G, et al. Association of atrial tissue fibrosis identified by delayed enhancement MRI and atrial fibrillation catheter ablation: the DECAAF study. JAMA. 2014;311:498-506.

32. Chen LY, Leening MJ, Norby FL, et al. Carotid intimamedia thickness and arterial stiffness and the risk of atrial fibrillation: the atherosclerosis risk in communities (ARIC) study, multi-ethnic study of atherosclerosis (MESA), and the Rotterdam study. JAm HeartAssoc. 2016;5:e2907.

33. Cui R, Yamagishi K, Muraki I, et al. Association between markers of arterial stiffness and atrial fibrillation in the circulatory risk in communities study (CIRCS). Atherosclerosis. 2017;263:244-8.

34. Kjeldsen SE, Aksnes TA, Wachtell K, Okin PM. Arterial stiffness predicts incident atrial fibrillation in the Framingham heart study: a mechanistic contribution in people with high blood pressure or history of hypertension. Hypertension. 2016;68:555-7.

35. Fumagalli S, Gabbai D, Nreu B, et al. Age, left atrial dimension and arterial stiffness after external cardioversion of atrial fibrillation. A vascular component in arrhythmia maintenance? Results from a preliminary study. Aging Clin Exp Res. 2014;26:327-30.

36. Lau DH, Middeldorp ME, Brooks AG, et al. Aortic stiffness in lone atrial fibrillation: a novel risk factor for arrhythmia recurrence. PLoS One. 2013;8:e76776.

37. Kizilirmak F, Guler GB, Guler E, et al. Impact of aortic stiffness on the frequency of paroxysmal atrial fibrillation recurrences. Acta Cardiol. 2015;70:414-21.

38. Marino PN, Degiovanni A, Baduena L, et al. Non-invasively estimated left atrial stiffness is associated with short-term recurrence of atrial fibrillation after electrical cardioversion. J Cardiol. 2017;69:731-8.

39. Chirinos JA, Segers P, Hughes T, Townsend R. Large-artery stiffness in health and disease: JACC state-of-the-art review. JAm Coll Cardiol. 2019;74:1237-63.

40. Lyle AN, Raaz U. Killing me unsoftly: causes and mechanisms of arterial stiffness. Arterioscler Thromb Vasc Biol. 2017;37:e1-el1.

41. Palombo C, Kozakova M. Arterial stiffness, atherosclerosis and cardiovascular risk: pathophysiologic mechanisms and emerging clinical indications. Vascul Pharmacol. 2016;77:1-7.

42. Spartano NL, Augustine J, Lefferts W, et al. Arterial stiffness as a noninvasive tissue biomarker of cardiac target organ damage. Curr Biomark Find. 2014;4:23-34.

43. FumagalliS, Migliorini M, PupoS, etal. Arterial stiffness and left ventricular performance in elderly patients with persistent atrial fibrillation. Aging Clin Exp Res. 2018;30:1403-8.

44. Fumagalli S, Giannini I, Pupo S, et al. Atrial fibrillation after electrical cardioversion in elderly patients: a role for arterial stiffness? Results from a preliminary study. Aging Clin Exp Res. 2016;28:1273-7.

45. Shen MJ, Arora R, Jalife J. Atrial myopathy. JACC Basic Transl Sci. 2019;4:640-54.

46. FaustinoA,ProvidênciaR, BarraS, etal. Whichmethod ofleft atrium size quantification is the most accurate to recognize thromboembolic risk in patients with non-valvular atrial fibrillation? Cardiovasc Ultrasound. 2014;12:28.

47. Njoku A, Kannabhiran M, Arora R, et al. Left atrial volume predicts atrial fibrillation recurrence after radiofrequency ablation: a meta-analysis. Europace. 2018;20:33-42.

48. Shin S-H, Park M-Y, Oh W-J, et al. Left atrial volume is a predictor of atrial fibrillation recurrence after catheter ablation. JAm SocEchocardiogr. 2008;21:697-702.

49. Lantelme P, Laurent S, Besnard C, et al. Arterial stiffness is associated with left atrial size in hypertensive patients. Arch Cardiovasc Dis. 2008;101:35-40.

50. Drager LF, Bortolotto LA, Pedrosa RP, Krieger EM, LorenziFilho G. Left atrial diameter is independently associated with arterial stiffness in patients with obstructive sleep apnea: potential implications for atrial fibrillation. Int $\mathrm{J}$ Cardiol. 2010;144:257-9.

51. Tsang TS, Barnes ME, Abhayaratna WP, et al. Effects of quinapril on left atrial structural remodeling and arterial stiffness. Am J Cardiol. 2006;97:916-20. 